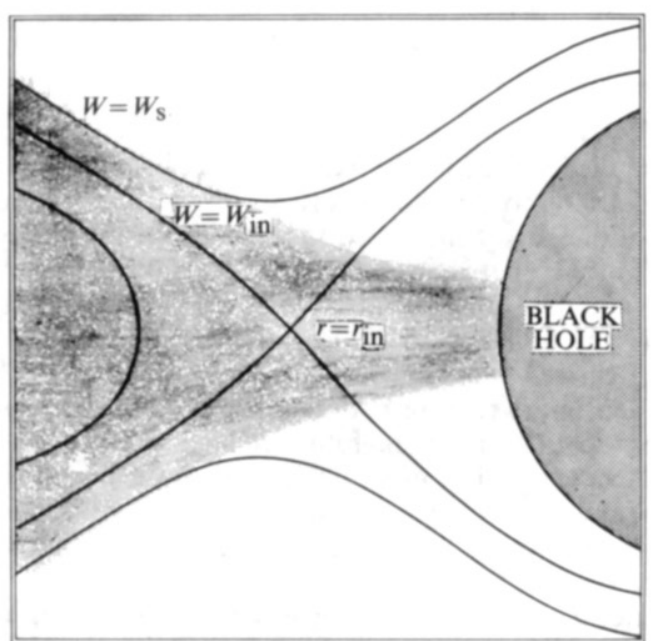

Fig. 1 Equipotential surfaces close to the inner edge of the disk.

law has $m=2, n=0$. Non-standard viscosity laws have $m<2$, $n=0$.

A recently discovered ${ }^{2}$ mass loss mechanism which operates close to the inner edges of accretion disks orbiting black holes removes both thermal and secular instabilities. There are no instabilities even in the extreme case when the ratio of radiation pressure to the total pressure, $\beta=1$, and when the ShakuraSunyaev $\alpha$-viscosity parameter, $\alpha=1$. The conclusion holds for both standard and non-standard viscosity laws.

Piran's arguments and his stability conditions are used here. After concluding that there is no combination of any known cooling mechanism with the standard viscosity law which gives rise to a stable inner disk region (radiation pressure and electron scattering-dominated), Piran remarks that a secondary wind escaping from the disk surface would have a stabilizing effect. He admits, however, that generally this wind would be too small to change the main conclusion significantly. Nevertheless, he gives explicit forms of the necessary and sufficient stability conditions for thermal and secular stability of a disk with a secondary wind. The conditions are general and valid not only for winds, but for any mass loss from the surface. The mass loss is expressed by

$$
\dot{M} \sim U^{s} H^{r}
$$

and the stability conditions yield

$$
\begin{gathered}
(8 s-3 r)+(51 s-9 r) \beta-(3 s+12 r) \beta^{2}-X^{2}(m-k)>0 \\
r(n+1-l)-s(m-k)>0 \\
{[2 m+8(n+1)]+[9 m+51(n+1)] \beta-3[m+n+1] \beta^{2}>0} \\
{[s m-(n+1) r]-Y^{2}[m l-(n+1) k]>0}
\end{gathered}
$$

The explicit form of $X^{2}$ and $Y^{2}$ is not relevant here.

General relativistic effects in the gravitational field of a black hole cause a characteristic behaviour of the equipotential surfaces close to the inner edge of an accretion disk: one of the equipotentials, $W=W_{\text {in }}$, crosses itself on the edge (see Fig. 1). If the surface of the disk, $W \approx W_{\text {s }}$, slightly overflows the critical equipotential $W=W_{\text {in }}$, then mechanical equilibrium is slightly destroyed, causing mass loss. The physical picture is analogous to the case of Roche lobe overflow in close binaries.

Abramowicz, Calvani and Nobili ${ }^{3}$ give expressions for the mass loss rate and efficiency of the cooling mechanism connected with the mass loss assuming only mechanical, not thermal, equilibrium. Their equations (5.19) and (5.13) valid close to the inner edge of the disk, that is for $r \approx r_{\text {in }}$, read

$$
\begin{gathered}
\dot{M} \sim U H \\
Q^{-} \sim U H^{3}
\end{gathered}
$$

Thus, for this particular mass loss mechanism $s=1, r=1, l=1$ and $k=3$ (for $r \approx r_{\text {in }}$ ).

This result, when combined with the general stability criteria of equation (5), proves that accretion disks orbiting black holes are thermally and secularly stable close to their inner edges. This is true for both standard and non-standard viscosity laws and it does not depend on any particular value of $\alpha$ or $\beta$.

Note that the stabilizing mechanism is purely general relativistic in its origin. This may be the only known situation when a general relativistic effect stabilizes a configuration, especially a rotating one.

The physics beyond this formal proof is simple: consider a small, purely thermal (that is with $U=$ constant) perturbation which produces an excess heat $\left(Q^{+}>Q^{-}\right)$. If the excess heat cannot be removed from the disk, the disk expands. This causes increased Roche lobe overflow: the location of the Roche lobe depends only on the angular momentum at $r=r_{\text {in }}$ and does not change during the expansion. Because the cooling increases very strongly with the increasing overflow and the heating does not, the disk reaches the state $Q^{-}=Q^{+}$and achieves thermal stability.

More detailed treatment of this phenomenon will be published elsewhere where I shall discuss the radial size of the region, $r_{\text {in }} \leqslant r \leqslant r_{*}$, affected by this stabilizing mechanism. The size, of course, depends strongly on the particular situation. A rough estimate gives $r_{*}>H\left(r_{\text {in }}\right) f\left(r_{*}\right) \approx$ few $r_{\text {in }}$, where $f=$ (thermal time scale/free-fall time scale). For the supermassiveblack-hole case this is enough to stabilize the whole inner (radiation pressure- and electron scattering-dominated) region of the disk.

I thank Geoffrey Bath and Tsvi Piran for helpful discussions.

Received 10 August; accepted 18 September 1981.

1. Piran, T. Astrophys. J. 221, 652 (1978).

2. Abramowicz M. Jaroszý́ski M. \& Sikora M. Astr. Astrophys. 63, 221 (1978).

3. Abramowicz, M. A., Calvani, M. \& Nobili, L. Astrophys. J. 242, 772 (1980).

\section{Milliarcsecond structure of BL Lac during outburst}

\author{
R. L. Mutel*, H. D. Aller $\dagger$ \& R. B. Phillips $\ddagger$ \\ * Department of Physics and Astronomy, The University of Iowa, \\ Iowa City, Iowa 52242, USA \\ + Department of Astronomy, The University of Michigan, Ann \\ Arbor, Michigan 48109, USA \\ $\ddagger$ Department of Physics, The University of Kansas, Lawrence, \\ Kansas 55044, USA
}

The well known object BL Lacerate is the prototype of a class of compact extragalactic sources which display rapid flux and polarization changes at radio and optical wavelengths and a nearly featureless optical spectrum'. After a period of relative quiescence, BL Lac has recently undergone a violent outburst at radio wavelengths, accompanied by rapid changes in degree of polarization and position angle (Fig. 1) ${ }^{2,3}$. For a better understanding of the physical mechanism responsible for the variable emission, we have mapped the radio structure at 5 and $10.6 \mathrm{GHz}$ at three epochs during the large flux outburst of 1980 with an intercontinental VLBI array using telescopes in Bonn, West Germany; Green Bank, West Virginia; Westford, Massachusetts; Fort Davis, Texas; and Owens Valley, California. The synthesized beam had a resolution of about $1.0 \mathrm{~m}$ arc $\mathrm{s}$ at $\lambda 6 \mathrm{~cm}$ and $0.5 \mathrm{~m}$ arc $s$ at $\lambda 2.8 \mathrm{~cm}$, corresponding to linear sizes of 5.9 and 2.9 lyr (light year) at the source (using a redshift ${ }^{4}$ of 0.0695 and a Hubble constant of $55 \mathrm{~km} \mathrm{~s}^{-1} \mathrm{Mpc}^{-1}$ ). We show here that comparison of the size and flux density of the core component with the flux history provides evidence for relativistic beaming effects, independent of detailed model considerations. The flux densities shown in Fig. 1 were obtained at the University of Michigan radio observatory.

The observations were made at three epochs: 1980.41 $(\lambda 6 \mathrm{~cm}), 1980.73(\lambda 6 \mathrm{~cm})$ and $1980.93(\lambda 2.8 \mathrm{~cm})$. During each epoch, one telescope had a technical failure so that only four telescopes were used in each mapping analysis. The telescopes used the MKII VLBI recording scheme ${ }^{5}$ and were equipped 


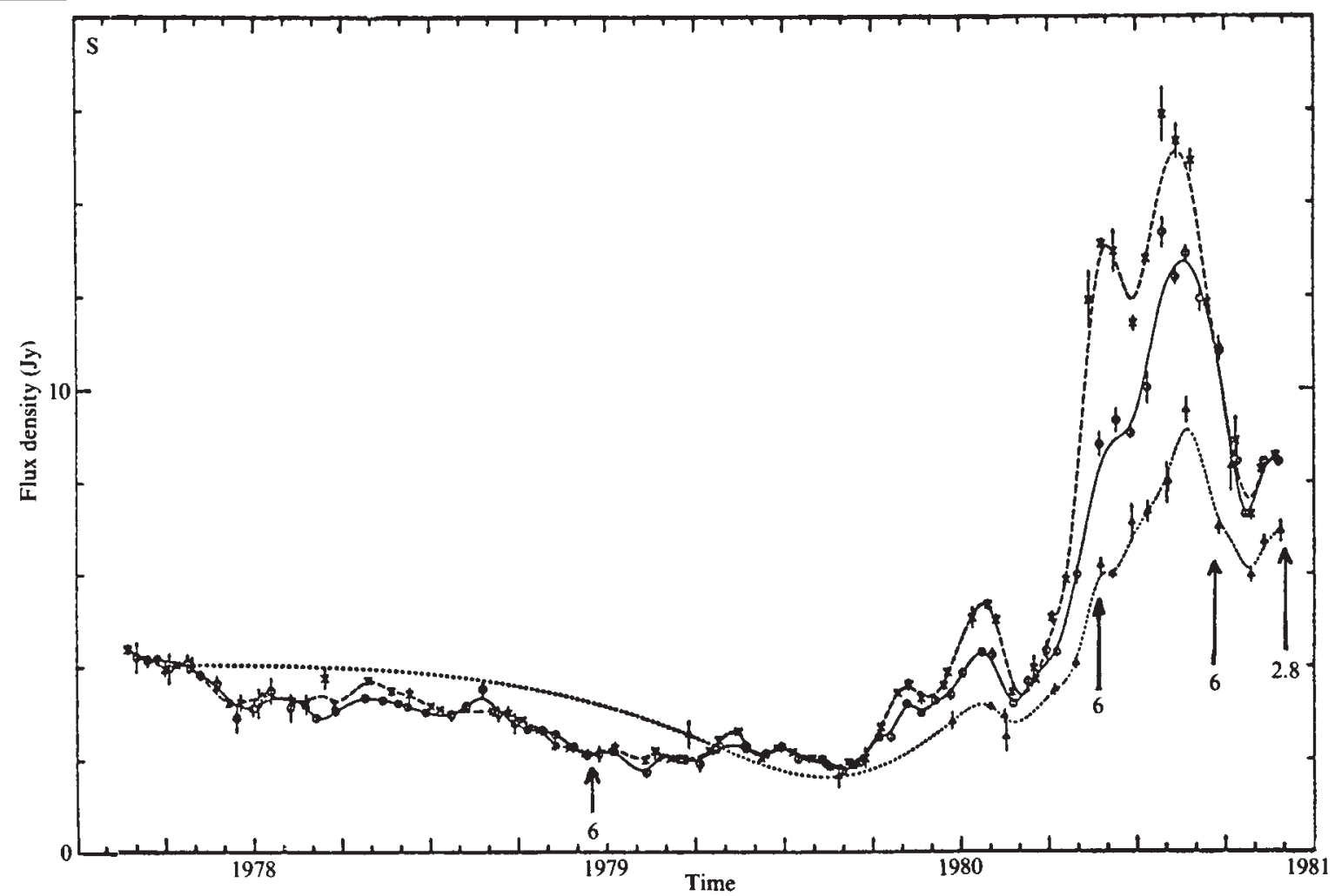

Fig. 1 Flux density compared with time at $4.8 \mathrm{GHz}(\Lambda \cdots \wedge \cdots), 8.0 \mathrm{GHz}(--)$ and $14.5 \mathrm{GHz}(\times--\times--)$. Data points are 2 -week averages. Epochs of VLBI observations are shown by arrows where the number indicates wavelength of each observations.

with maser time standards. All observations were made using left circular polarization and a receiving bandwidth of $2 \mathrm{MHz}$. The data were correlated using the Caltech/JPL VLBI correlator. The mapping was done at the University of lowa using a hybrid algorithm ${ }^{6}$ utilizing closure phase. For all three epochs, the resulting fits to the data were very good, and the maps are thought to be reliable to the $5 \%$ contour level. An example of the visibility data and corresponding hybrid model fit is given in Fig. 2 for the 1980.93 data at $\lambda 2.8-\mathrm{cm}$ wavelength.

The $\lambda 2.8-\mathrm{cm}$ hybrid map is shown in Fig. $3 a$, while the two $\lambda$ $6-\mathrm{cm}$ maps are shown in Fig. $4 a, b$. In each case, the restoring beam has been chosen by fitting an elliptical gaussian to the main beam of the array power pattern, which is obtained by Fourier transforming the transfer function for each observation. A remarkable aspect of the structure at all three epochs which is not apparrent on the hybrid maps is the extreme narrowness of the source. For both $\lambda 6-\mathrm{cm}$ observations, the source was completely unresolved when the baselines were aligned approximately east-west. The $\lambda 2.8-\mathrm{cm}$ data also show this effect, although there is some east-west resolution on the transatlantic baselines. This dominant north-south structure has been seen with more limited VLBI measurements ${ }^{7-9}$ as early as 1971 and apparently indicates that there is an alignment mechanism in the central source which is stable compared with the time scale of individual flux outbursts.

To clarify the detailed north-south structure, we have shown one-dimensional profiles of brightness temperature along a position angle of $0^{\circ}$ for each map. Figure $2 b$ shows the $\lambda 2.8-\mathrm{cm}$ profile, while Fig. 5 shows the $\lambda 6-\mathrm{cm}$ profiles at both epochs. In addition, we have shown the north-south brightness profile for the three-component gaussian model of Pearson and Readhead ${ }^{10}$ based on data taken at $\lambda 6-\mathrm{cm}$ in 1978 December when BL Lac was dormant (see Fig. 1). To compare brightness temperatures in a uniform way, we have used the same restoring beam for all three $\lambda 6-\mathrm{cm}$ profiles. This beam is too small for the December 1978 model (because only US baselines were used) so that the detailed structure of the components is probably unreliable. We have also convolved our data with the same restoring beam of Pearson and Readhead and find no evidence of the southerly component extending $\sim 10 \mathrm{~m}$ arcs $\mathrm{s}(60 \mathrm{lyr})$ seen in their 1978 map.

Comparison of the $\lambda 2.8$ map (Fig. 3 ) with the flux history data of Fig. 1 shows that the source is much larger than would be expected from simple light travel time arguments. The current outburst can be dated from $\sim 1979.8$ when the total flux density is $\sim 2 \mathrm{Jy}$ at $\lambda 6 \mathrm{~cm}$ and $\lambda 2.8 \mathrm{~cm}$. Without relativistic effects, the maximum source size in $\sim 1980.9$ would be 1.1 lyr, whereas the north-south extent of the $\lambda 2.8-\mathrm{cm}$ map is $\sim 4.5 \mathrm{lyr}$. This discrepancy could be understood in several ways: (1) simultaneous, independent brightening of causally unconnected regions; (2) activation by a single 'trigger' mechanism located approximately midway between two distant emitters; or (3) relativistic beaming effects causing a time 'speed-up' in the observed flux changes. Explanation (1) seems very improbable, particularly considering the 3-yr dormant period which preceded the outburst, while explanation (2) requires a highly specialized geometry.

The relativistic beam model ${ }^{11}$ has been widely used to explain the properties of compact radio sources ${ }^{12-14}$. The model consists of a central object with two opposed particle beams moving with relativistic bulk velocities. If the line of sight to the observer is nearly parallel to the beam axis, he will see the forward beam Doppler-boosted in intensity. The true angular source size is the apparent size as observed in the VLBI data, but the flux variations are observed 'speeded up' by the Lorentz factor, which in the present case is $\gamma \sim 4$.

A further argument supporting relativistic beaming in this source is the anomalously high brightness temperatures implied by the flux density variations. In the absence of relativistic effects, the inferred brightness temperature of the core component would be at least $10^{13} \mathrm{~K}$ based on the light travel size. This violates the inverse Compton limit of $T_{\mathrm{B}} \leqslant 10^{12} \mathrm{~K}$ for an opaque synchrotron emitter. On the other hand, the observed brightness temperatures (based on the actual measured angular sizes) are $T_{\mathrm{B}} \leqslant 3 \times 10^{11} \mathrm{~K}$ for all three observations.

The $\lambda 6-\mathrm{cm}$ maps do not have sufficient angular resolution to show the details of the evolution of the jet during the outburst. However, comparison of the 1980.41 and 1980.73 brightness 

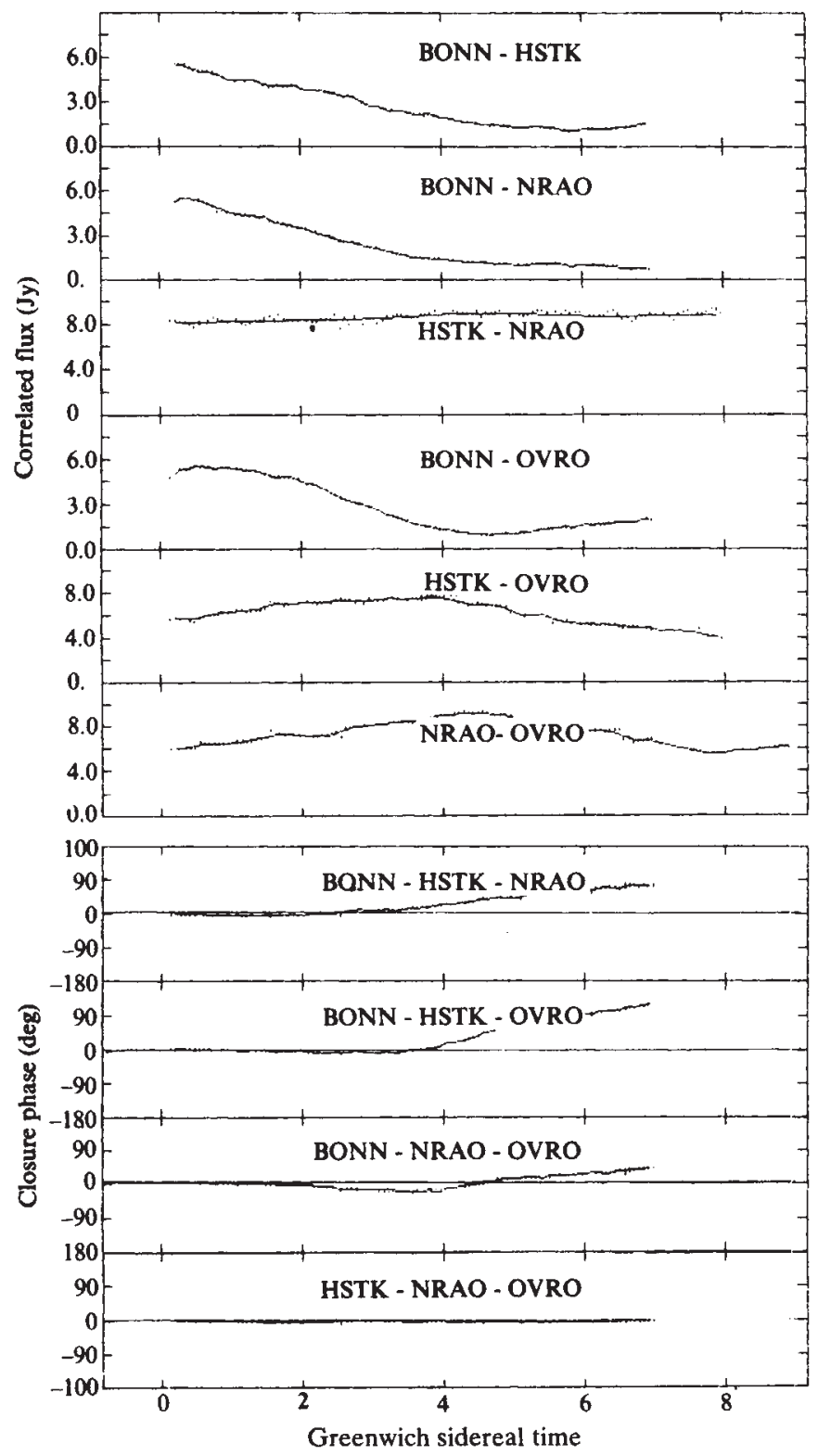

Fig. 2 Four-min averages of fringe amplitude and closure phase for the 1980.93 data at $10.6 \mathrm{GHz}$. Also shown is the fit from the hybrid map shown in Fig. $3 a$.
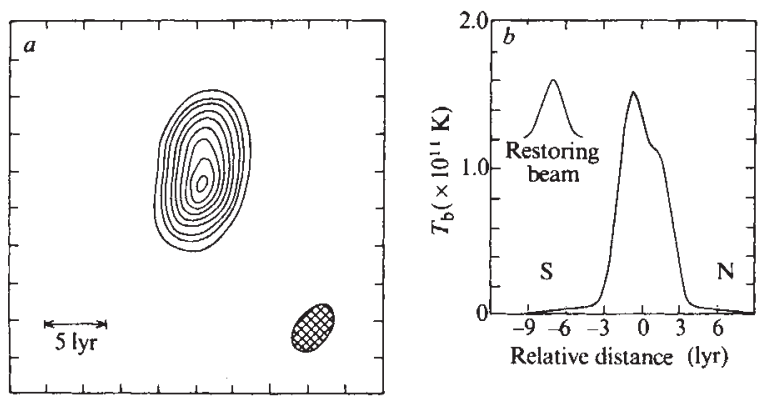

Fig. $3 a$, Hybrid map of BL Lac at $10.6 \mathrm{GHz}$, epoch 1980.93 . Contours are $5,15,25,35,45,55,65,75,85$ and $95 \%$ of peak flux. $b$, One-dimensional profile of peak brightness temperature along position angle $0^{\circ}$ in map in $a$.

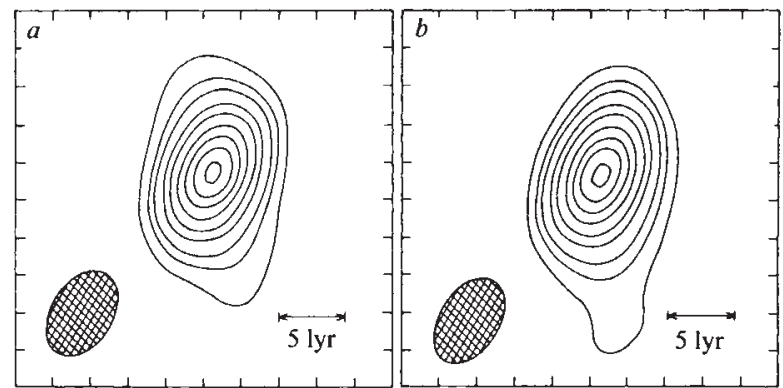

Fig. $4 a$, Hybrid map of BL Lac at $5 \mathrm{GHz}$, epoch 1980.41 . Contours as in Fig. 3a. b, Epoch 1980.73, same as in $a$.

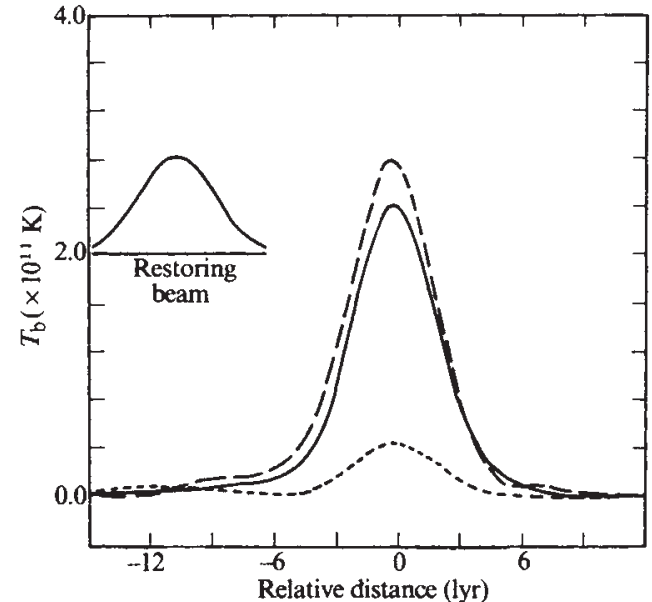

Fig. 5 One dimensional profiles of peak brightness temperature along position angle $0^{\circ}$ for $5 \mathrm{GHz}$ maps at three epochs. Dotted line is epoch 1978.93, solid line is epoch 1980.41 and dashed line is epoch 1980.73. Note that the relative position between epochs can not be determined from the VLBI data and is not necessarily as shown here. Also the same restoring beam has been used for all three epochs. This over-resolves the 1978.93 data (see text).

profiles (Fig. 5) show that there has been a small, but definite increase in the core size, of $\sim 0.5 \mathrm{lyr}$. This is actually a lower limit, as the true size has been convolved with a restoring beam whose dimensions are comparable with the core size. In addition, all three profiles show a weak, extended southerly component. The detailed nature of this component is uncertain because of the limited dynamic range of the maps. However, we have found differences in the closure phase on identical baseline triangles in the comparison of 1980.41 and 1980.72 data which we are confident are caused by changes in the extended southerly structure. The extended structure is not seen on the $\lambda$ $2.8-\mathrm{cm}$ profile, indicating that perhaps the extended emission has become optically thin between $\lambda 6$ and $\lambda 2.8 \mathrm{~cm}$.

We thank the staffs of the five observatories for assistance with the observations. The work was supported in part by NSF grants AST-79-10128 and AST-78-24192.

Received 9 March; accepted 2 October 1981

1. Stein, W. A. in Pittsburgh Conf. on BL Objects (University of Pittsburgh, Pennsylvania, 1978)

2. Aller, M. F., Aller, H. D. \& Hodge, P. E. Bull. Am. astr. Soc. 12, 803 (1981)

3. Aller, H. D., Aller, M. F. \& Hodge, P. E Astr I. 86, 325 (1981)

4. Miller, J. S. French, H. B. \& Hawley, S. A Astrophys, J. Lett 219, L85 (1978)

5. Clark, B. G. IEEE 61, 1242 (1973).

6. Readhead, A. C. S. \& Wilkinson, P. N. Astrophys. J. 223, 25 (1978)

7. Clark, B. G. et al. Astrophys. J. Lett. 182, L57 (1973)

8. Kellermann, K. I. et al. Astrophys. J. 211, $658(1977)$

9. Shaffer, D. B. in Pittsburgh Conf. on BL Objects (University of Pittsburgh, Pennsylvania, 1978).

10. Pearson, T. J. \& Readhead, A. C. S. Astrophys. J. 248, 671 (1981)

11. Biandford, R. D. \& Königl, A. Astrophys. J. 232, 34 (1979)

12. Readhead, A. C. S. Phys. Scr. 21, 662 (1980)

13. Scheuer, P. A. G. \& Readhead, A. C. S. Nature 277, 182 (1979).

14. Kellermann, K. I. \& Pauliny-Toth, I. I. K. A. Rev. Astr. Astrophys. (in the press), 